\title{
The mesoSPIM initiative: open-source light-sheet mesoscopes for imaging in cleared tissue
}

Fabian F. Voigt ${ }^{1,2}$, Daniel Kirschenbaum ${ }^{3}$, Evgenia Platonova ${ }^{4}$, Stéphane Pagès ${ }^{5,6}$, Robert A. A. Campbell ${ }^{7}$, Rahel Kästli ${ }^{1,2}$, Martina Schaettin ${ }^{8,2}$, Ladan Egolf ${ }^{1,2}$, Alexander van der Bourg ${ }^{1,2}$, Philipp Bethge ${ }^{1,2}$, Karen Haenraets ${ }^{9,2}$, Noémie Frézel $^{9,2}$, Thomas Topilko ${ }^{10}$, Paola Perin ${ }^{11}$, Daniel Hillier $^{12,13}$, Sven Hildebrand ${ }^{15}$, Anna Schueth ${ }^{15}$, Alard Roebroeck ${ }^{15}$, Botond Roska ${ }^{16,17}$, Esther Stoeckli $^{8,2}$, Roberto Pizzala ${ }^{18}$, Nicolas Renier ${ }^{9}$, Hanns Ulrich Zeilhofer ${ }^{9,2}$, Theofanis Karayannis ${ }^{1,2}$, Urs Ziegler ${ }^{4}$, Laura Batti ${ }^{5}$, Anthony Holtmaat ${ }^{6}$, Christian Lüscher ${ }^{6,19}$, Adriano Aguzzi $^{3}$, and Fritjof Helmchen ${ }^{1,2}$

${ }^{1}$ Brain Research Institute, University of Zurich, ${ }^{2}$ Neuroscience Center Zurich, University of Zurich and ETH Zurich, ${ }^{3}$ University Hospital Zurich, ${ }^{4}$ Center for Microscopy and Image Analysis, University of Zurich, ${ }^{5}$ Wyss Center for Bio- and Neuroengineering, Geneva, ${ }^{6}$ Department of Basic Neurosciences, University of Geneva, ${ }^{7}$ Sainsbury Wellcome Centre for Neural Circuits and Behaviour, London, ${ }^{8}$ Institute of Molecular Life Sciences, University of Zurich, ${ }^{9}$ Institute of Pharmacology and Toxicology, University of Zurich, ${ }^{10}$ ICM - Brain \& Spine Institute, Paris, ${ }^{11}$ Department of Brain and Behavioural Sciences, University of Pavia, ${ }^{12}$ Hungarian Academy of Sciences Research Centre for Natural Sciences, ${ }^{13}$ Faculty of Information Technology and Bionics, Pazmany Peter Catholic University, ${ }^{15}$ Faculty of Psychology \& Neuroscience, Maastricht University, ${ }^{16}$ Friedrich Miescher Institute Basel, ${ }^{17}$ Institute of Molecular and Clinical Ophthalmology Basel, ${ }^{18}$ Department of Molecular Medicine, University of Pavia, ${ }^{19}$ Clinic of Neurology, Dept. of Clinical Neurosciences, Geneva University Hospital, 
Over the course of the past decade, tissue clearing methods have reached a high level of sophistication with a wide variety of approaches now available ${ }^{1}$. To image large cleared samples, light-sheet microscopes have proven to be ideal due to their excellent optical sectioning capability in transparent tissue ${ }^{2}$. Such instruments have recently seen extensive technological and commercial development. However, despite this progress, the community is lacking instruments capable of exploring large samples with near-isotropic resolution within minutes. Here, we introduce the mesoscale selective plane-illumination microscopy (mesoSPIM) initiative, an open-hardware project that provides researchers with instructions and software to easily build and operate lightsheet microscopes for centimeter-sized cleared samples (http://www.mesospim.org).

A wide range of commercial light-sheet microscopes have become available since the invention of $\mathrm{SPIM}^{3}$, most of them optimized towards time-lapse imaging in transparent developing embryos or - if designed for cleared tissue - tailored for only a narrow selection of clearing techniques and immersion media. To overcome these limitations, we set out to design a modular light-sheet mesoscope (Fig. 1a) that combines simple and versatile sample handling with large fields-of-view (FOV) of 2-20 mm. Large-FOV light-sheet microscopes typically suffer from non-uniform axial resolution due to the varying thickness of the light sheet (Fig. 1b). To address this issue, we use a tunable lens to shift the excitation beam waist through the sample in synchrony with the rolling shutter of the camera, a method called 'axially scanned light-sheet microscopy' (ASLM) ${ }^{4}$ (Fig. 1b). For whole mouse brains $\left(\approx 1 \mathrm{~cm}^{3}\right)$, typical datasets are isotropic (6.5 $\mu \mathrm{m}$ sampling), small (12-16 GB), and they are recorded quickly (7-8 minutes) with minimal shadow artifacts. Together with standardized quick-exchange holders, these features allow for the fast screening of samples. With a travel range of $44 \times 44 \times 100 \mathrm{~mm}$, very large samples such as a whole mouse central nervous system can be imaged. After acquiring overview datasets, users can zoom in and record multidimensional 
data at higher resolution by mosaic acquisitions, for example revealing cellular distribution and long-range axonal projections of Purkinje cells in the mouse cerebellum (Fig. 1c-e) or fine neurites in the developing nervous system of a chick embryo (Fig. 1f). The instrument has been tested in combination with all common clearing methods ranging from CLARITY ${ }^{5}, \mathrm{CUBIC}^{6}$ to organic solvent approaches such as $\mathrm{iDISCO}^{7}$ and $\mathrm{BABB}^{2}$. Due to the modular design of the mesoSPIM, switching between different imaging media can be done in less than a minute. The mesoSPIM setup, performance characterization, and example applications are presented with detail in Supplementary Notes including Supplementary Figures 1-30. Inspired by the openSPIM ${ }^{8}$ and openSPIN ${ }^{9}$ projects, documentation for the mesoSPIM hardware and software (written in Python) is freely available (https://github.com/mesoSPIM). Currently, there are 5 mesoSPIM setups in operation across Europe. The mesoSPIM is the ideal instrument to quickly bridge scales from the $\mu \mathrm{m}$ - to the cm-level, and serves as an excellent tool for detailed three-dimensional anatomical investigations in neuroscience and developmental biology.

\section{Code availability}

The mesoSPIM software and documentation are available on Github (https://github.com/mesoSPIM). mesoSPIM-control is licensed under the GNU General Public License v3.0 (GPL v3). 


\section{Acknowledgements}

This work was supported by grants from the Swiss National Science Foundation (310030B_170269, F.H.; 31003A-153448, 31003A_173125, CRSII3_154453, and NCCR

Synapsy 51NF40-158776, A.H.), the European Research Council (ERC Advanced Grant BRAINCOMPATH, project 670757; F.H.), ERC Starting Grant (MULTICONNECT, project 639938; AR), the Dutch science foundation (NWO VIDI Grant, project 14637; AR), and a gift from a private foundation with public interest through the International Foundation for Research in Paraplegia (A.H. and S.P.) In addition, we would like to thank Dubravka Göckeritz-Dujmovic and Sandrine Bichet for help with sample preparation and Martin Wieckhorst for help with custom electronics.

\section{Author information - Contributions}

F.F.V.: planned the project, designed the microscope, wrote control software and documentation, set up several mesoSPIM instruments, coordinated the mesoSPIM initiative, imaged samples, analyzed data and wrote the manuscript.

R.K.,M.S, L.E., A. v.d. B, K. H., N.F., T.T., N.R., H-U. Z., T.K., P.P., R.P., D.H., B.R, S.H., A.S., A.R.: Prepared samples for imaging.

S.P., E. P., D.K., R.A.A.C, F.M., L.B. A.H., C.L., A.A.: Set up mesoSPIM instruments.

F.H. planned the project and wrote the manuscript.

Competing interests: None to disclose.

\section{Corresponding author:}

Correspondence to Fabian F. Voigt. voigt@ hifo.uzh.ch 


\section{References}

1 Richardson, D. S. \& Lichtman, J. W. Clarifying Tissue Clearing. Cell 162, 246-257, doi:10.1016/j.cell.2015.06.067 (2015).

2 Dodt, H.-U. et al. Ultramicroscopy: three-dimensional visualization of neuronal networks in the whole mouse brain. Nature Methods 4, 331-336, doi:10.1038/nmeth1036 (2007).

3 Huisken, J., Swoger, J., Del Bene, F., Wittbrodt, J. \& Stelzer, E. H. Optical sectioning deep inside live embryos by selective plane illumination microscopy. Science 305, 10071009, doi:10.1126/science.1100035 (2004).

4 Dean, K. M., Roudot, P., Welf, E. S., Danuser, G. \& Fiolka, R. Deconvolution-free Subcellular Imaging with Axially Swept Light Sheet Microscopy. Biophysical Journal 108, 2807-2815, doi:10.1016/j.bpj.2015.05.013 (2015).

5 Chung, K. et al. Structural and molecular interrogation of intact biological systems. Nature 497, 332-337 (2013).

6 Susaki, E. A. et al. Whole-Brain Imaging with Single-Cell Resolution Using Chemical Cocktails and Computational Analysis. Cell 157, 726 - 739 (2014).

7 Renier, N. et al. iDISCO: A Simple, Rapid Method to Immunolabel Large Tissue Samples for Volume Imaging. Cell 159, 896-910, doi:10.1016/j.cell.2014.10.010 (2014).

8 Pitrone, P. G. et al. OpenSPIM: an open-access light-sheet microscopy platform. Nat Methods 10, 598-599, doi:10.1038/nmeth.2507 (2013).

9 Gualda, E. J. et al. OpenSpinMicroscopy: an open-source integrated microscopy platform. Nat Methods 10, 599-600, doi:10.1038/nmeth.2508 (2013). 
bioRxiv preprint doi: https://doi.org/10.1101/577122; this version posted March 18, 2019. The copyright holder for this preprint (which was not

certified by peer review) is the author/funder, who has granted bioRxiv a license to display the preprint in perpetuity. It is made available under aCC-BY-ND 4.0 International license.

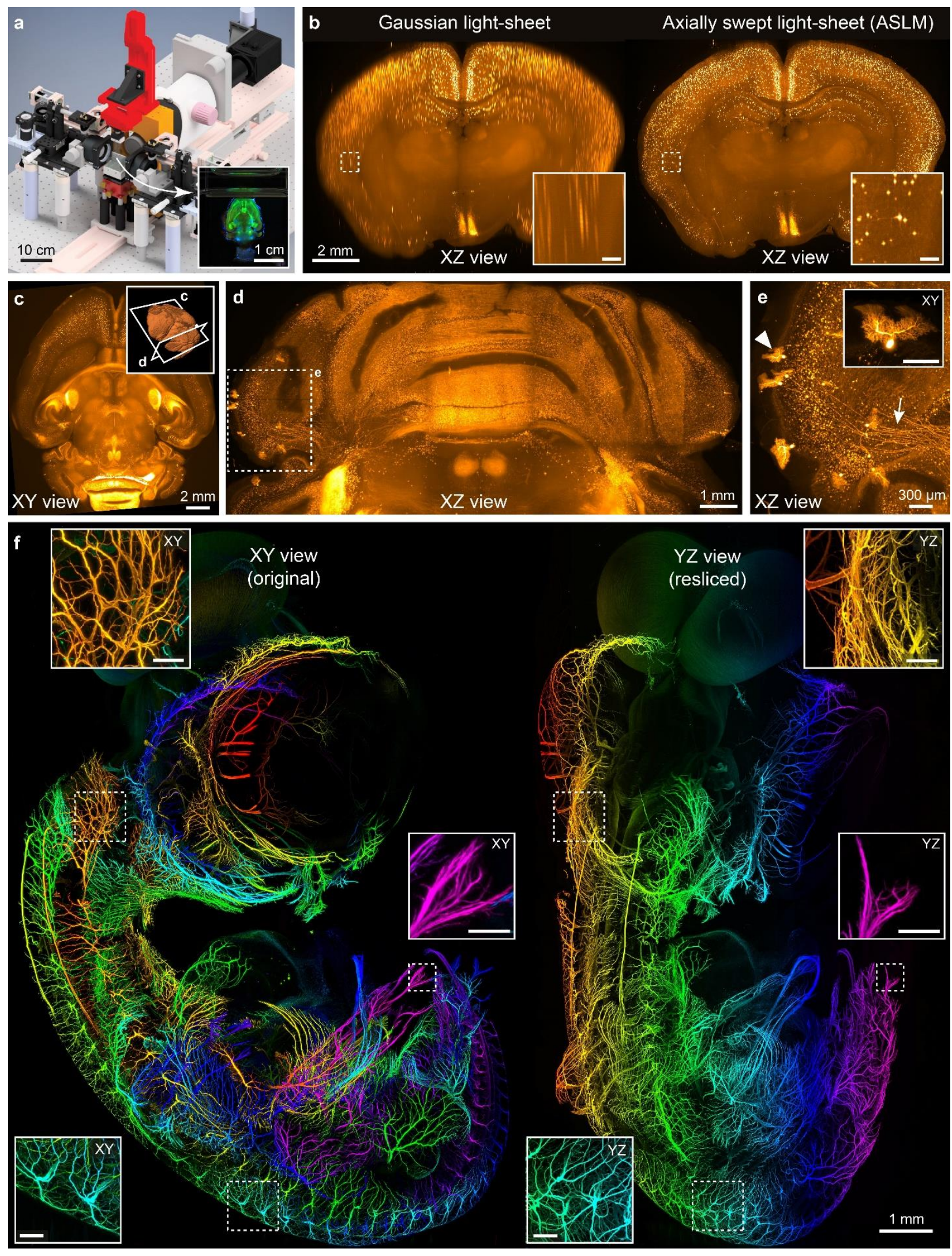


Figure 1: Example demonstrations of the mesoSPIM light-sheet mesoscope. a) Overview of the mesoSPIM instrument. Inset: Photograph of a Thy1-YFP mouse brain during image acquisition. b) Comparison of axial image quality achieved in a CLARITY-cleared VIPtdTomato mouse brain for scanning with a Gaussian beam (left) and for the axially swept lightsheet mode, ASLM ( right). Images are maximum intensity projections over $250-\mu \mathrm{m}$ range. c) Overview image of a CLARITY-cleared TPH2Cre-tdTomato mouse brain. Inset shows 3D orientation of views in c and d. d) XZ maximum intensity projection of a high-resolution dataset (4× zoom, range: $500 \mu \mathrm{m}$ ) taken in the cerebellum of the sample in c. e) Volume rendering of sparsely labeled Purkinje cells and their axonal projections (arrow). Inset: individual Purkinje cell (arrow head, scale bar: $200 \mu \mathrm{m}$ ). f) Depth-coded original XY (left) and resliced YZ (right) projections of a dataset taken from a 7-day old chicken embryo (neurofilament labeling) cleared using BABB. Throughout the dataset (acquired at $1.6 \times 1.6 \times 2 \mu \mathrm{m}^{3}$ sampling), neurites are visible in great detail. Because of the ASLM mode this same holds true for the original (transverse) and the resliced (axial) direction. The assignment of color to Z-position is similar for both the XY and YZ view. Scale bars of all insets: $200 \mu \mathrm{m}$. 